\title{
Ultrastructure of Capillaries in Mouse Periodontium
}

\author{
R. E. CORPRON, J. K. AVERY, A. P. MORAWA, and S. D. LEE \\ Departments of Pedodontics and Oral Biology, and The Dental Research Institute, \\ School of Dentistry, The University of Michigan, Ann Arbor, Michigan 48109, USA
}

Considering the cellular density of periodontium, its vascularity is abundant (PROVENZA ET AL, Oral Surg 13: 157-164, 1974) which reflects the presence of two peripherally located cellular layers of bone development and cemental deposition, processes which when active have high metabolic requirements. The microcirculatory bed of the periodontium serves functionally for the exchange of gases and nutrients at the cellular level (MajNo, Handbook of Physiological Circulation, Vol 3, 1965) .

The purpose of this investigation is to describe the distribution and ultrastructure of terminal ressels in the periodontium of mouse molars. Mandibular first molars with periodontium and surrounding alveolar bone were removed in block sections from 25- to 50-day-old Swiss-Webster mice under sodium amytal. Blocks were trimmed, fixed in one-half strength Karnovsky fixative, postfixed in osmic acid, dehydrated, embedded in epoxy resin, ${ }^{a}$ sectioned, doublestained with uranyl acetate and lead citrate, and evaluated with an electron microscope. ${ }^{b}$ Thick sections (1 micrometer) were stained with methylene blue and viewed with a light microscope to determine orientation of the tissue and location of the section.

Observations from 25- or 50-day-old mice did not vary significantly. Capillaries showed fingerlike projections that protruded into the lumen. The cytoplasm of the endothelial cells contained numerous microvesicles, scattered ribosomes, and small oval mitochondria. Occasional tight junctions appeared between endothelial cells lined completely by a distinct basement lamina but incompletely encircled by pericytes. Although central capillaries in the periodontium appeared devoid of fenestrations, fenestrated capillaries ap. peared peripherally and close to the osteoblastic layer. Pores appeared along flattened areas of the capillary wall as 300 to $500 \mathrm{~A}$ openings bridged by thin membranes continuous with either side of the endothelial cell.

Small peripheral arterioles appeared near alveolar bone or near cementum and displayed an incomplete muscular coating. Myoendothelial junctions (RHodin, J Ultrastruct Res 18: 181-223, 1967), were occasionally observed between the endothelial cells and investing smooth muscle.

These fenestrations may represent rapid path-

This investigation was supported, in part, by The Dental Research Institute Grant DE 02731 .

Received for publication July 8, 1975.

Accepted for publication September 17, 1975.

a Epon 812, Epoxy resin: Ernest F. Fullam Inc. Schenectady, NY.

${ }^{b}$ Hitachi Limited, Tokyo, Jap. ways of metabolites across endothelium to the periodontium where high metabolic requirements for growth or repair occur.
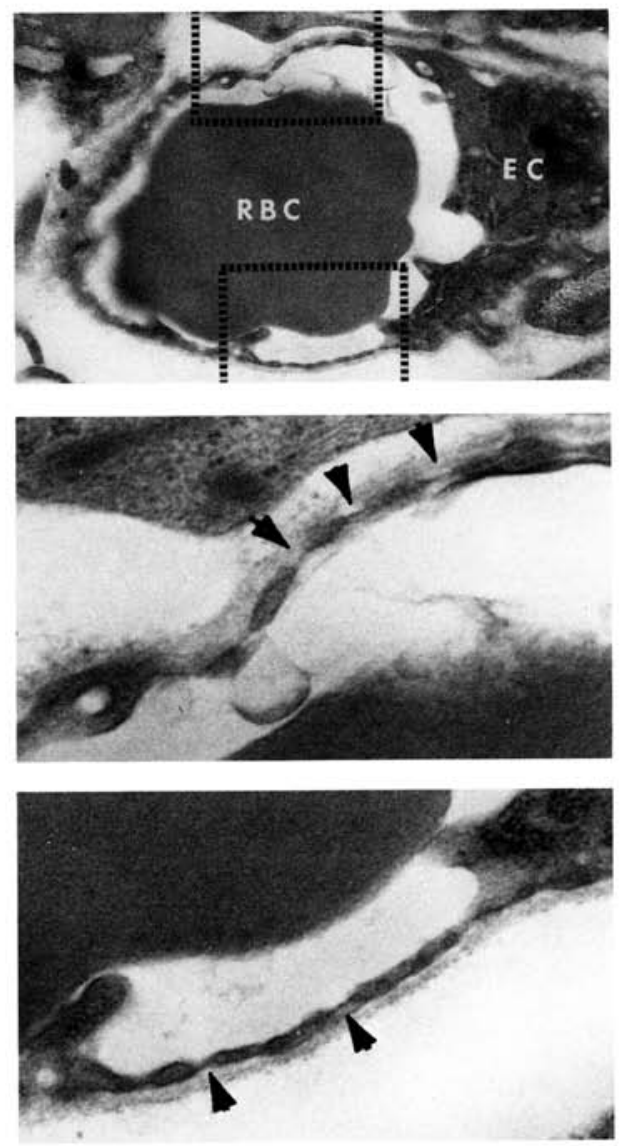

Top, small, fenestrated capillary with red blood cell (RBC) in lumen. Fenestrations appear along wall of endothelial cell (EC) away from nucleus (insets) (mag $\times 24,500)$. Center (top inset), arrows point to fenestrations (300 to $500 \mathrm{~A})(\mathrm{mag} \times 38,100)$. Bottom (bottom inset), arrows point to fenestrations in endothelial wall. Distinct basement lamina is visible below fenestrated capillary wall (mag $\times 38,100)$. 